\title{
Muon radiography for exploration of Mars geology
}

\author{
S. Kedar ${ }^{1}$, H. K. M. Tanaka ${ }^{2}$, C. J. Naudet ${ }^{1}$, C. E. Jones ${ }^{1}$, J. P. Plaut ${ }^{1}$, and F. H. Webb ${ }^{1}$ \\ ${ }^{1}$ Jet Propulsion Laboratory, California Institute of Technology, 4800 Oak Grove Dr., Pasadena, CA, USA \\ ${ }^{2}$ Earthquake Research Institute, University of Tokyo, Tokyo, Japan \\ Correspondence to: S. Kedar (sharon.kedar@jpl.nasa.gov)
}

Received: 31 August 2012 - Published in Geosci. Instrum. Method. Data Syst. Discuss.: 18 October 2012 Revised: 29 March 2013 - Accepted: 7 April 2013 - Published: 17 June 2013

\begin{abstract}
Muon radiography is a technique that uses naturally occurring showers of muons (penetrating particles generated by cosmic rays) to image the interior of large-scale geological structures in much the same way as standard X-ray radiography is used to image the interior of smaller objects. Recent developments and application of the technique to terrestrial volcanoes have demonstrated that a low-power, passive muon detector can peer deep into geological structures up to several kilometers in size, and provide crisp density profile images of their interior at ten meter scale resolution. Preliminary estimates of muon production on Mars indicate that the near horizontal Martian muon flux, which could be used for muon radiography, is as strong or stronger than that on Earth, making the technique suitable for exploration of numerous high priority geological targets on Mars. The high spatial resolution of muon radiography also makes the technique particularly suited for the discovery and delineation of Martian caverns, the most likely planetary environment for biological activity.

As a passive imaging technique, muon radiography uses the perpetually present background cosmic ray radiation as the energy source for probing the interior of structures from the surface of the planet. The passive nature of the measurements provides an opportunity for a low power and low data rate instrument for planetary exploration that could operate as a scientifically valuable primary or secondary instrument in a variety of settings, with minimal impact on the mission's other instruments and operation.
\end{abstract}

\section{Introduction}

Muon radiography was first applied for practical purposes as an imaging technique in the 1950 s by E. P. George to measure the overburden over a tunnel in Australia (George, 1955), and in the 1960s by Luis Alvarez in his famous attempt to discover hidden chambers in the Second Pyramid of Chephren in Giza (Alvarez et al., 1970). Although the concept has been around for decades, recent advances in detector technology and data processing have brought the technology to a point where it can be used reliably for subsurface imaging of geological structures. This was recently demonstrated in several terrestrial volcano imaging campaigns (Tanaka et al., $2010,2009 a, b, 2008$ ) that provided subsurface density profiles of unprecedented resolution (Fig. 1). The detected variations are of the order of a few percent in density contrast, making it a powerful tool for discriminating between hot rock and cold rock, rock and voids, and rock and water. Applying this technique to Martian geology is attractive for several reasons: (1) cosmic rays are ubiquitous and generate secondary muons in any atmosphere; (2) a preliminary Monte Carlo simulation of the cosmic ray muon production on the Martian surface by Tanaka (2007) shows that the technique is ripe for application on planetary bodies other than the Earth; (3) high energy muons are extremely penetrating, easily passing through hundreds of meters of rock; (4) detectors, as collectors of muons radiation, are passive in the sense that they use nature's cosmic rays and thus have significantly lower mass and power requirements than active imagers, such as radar, sonar or X-ray; (5) successful application of this technology on planetary bodies would bring new capabilities to future Solar System exploration missions by enabling highresolution imaging of the interior of planetary surface features with passive sensing of secondary cosmic ray muons, 


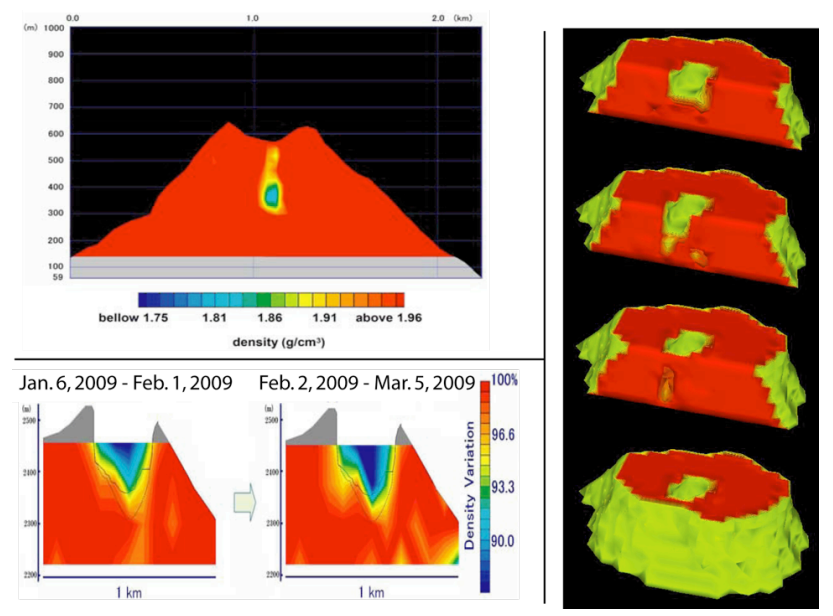

Fig. 1. Multiple examples of the resolving power of muon radiography. Top left: density profile of a terrestrial volcano obtained with secondary cosmic ray muons (Tanaka et al., 2009a). Bottom left: detected mass change inside a volcano using cosmic-ray muon radiography (Tanaka et al., 2009b). Right: vertical slices showing a 3-D profile of the interior of Mt. Asama constructed by Tanaka et al. (2010) using data from a muon radiography survey at multiple locations around the dome.

thereby providing new information about the planet's evolutionary state and history.

Cosmic rays are particles generated in outer space, usually of extra-solar origin. The primary cosmic rays reaching the outer edge of the Earth's atmosphere, and which create showers of particles that reach Earth's surface, are composed of $90 \%$ protons, $9 \%$ alpha particles and $1 \%$ heavier nuclei (Gaisser, 1990). Many are very high energy, but each of these primary constituents undergoes strong nuclear interactions and therefore none are sufficiently penetrating to be used directly for radiography of planetary surfaces. However, as the primary cosmic rays interact with matter in the atmosphere, they generate showers of secondary particles, which in turn can decay or create more showers. These extensive air showers (EAS) in the upper atmosphere generate many unstable mesons (hadronic subatomic particles including pions and kaons) that decay into muons. Muons have a long lifetime and a small cross section for interactions, so those with high energy and relativistic momentum can travel long distances before decaying. Most importantly, muons penetrate deep into rock (Fig. 2), enabling reconstruction of the 3-D internal structure of a geological feature from its muon absorption profile.

The muon generation rate, energy spectrum, and surface flux all depend on the atmosphere in which they are generated (Grieder et al., 2001). To design an experiment and a detector for muon radiography on a planetary surface, it is necessary to tailor the instrument requirements to the muon flux and energy distribution of near-horizontally traveling muons generated in the atmosphere of the body, given the mission

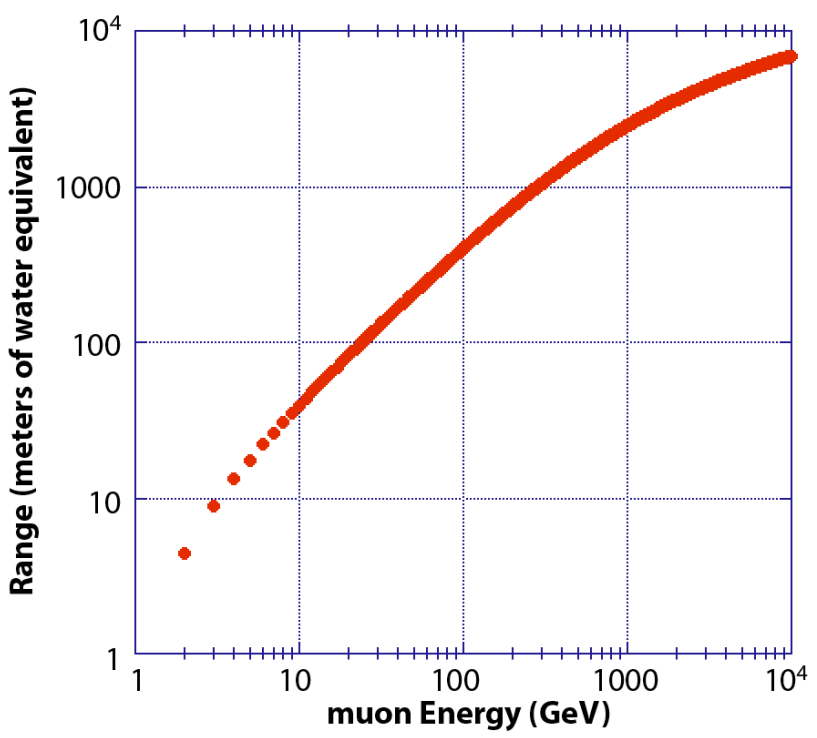

Fig. 2. The muon range in standard rock underground on Earth as a function of muon energy, expressed in meters of water equivalent (Reichenbacher and De Jong, 2007). The depth accessible to muon radiography depends upon the energy of the muons, the composition of the rock in question, the surface muon flux, target/detector geometry, detector size and efficiency, image resolution and measurement integration time.

objectives and duration. To determine whether the science requirements can be met, the horizontal muon flux at the Martian surface must be estimated. Particle production rates in any given atmosphere are simulated using a Monte Carlo approach accounting for the initial cosmic ray particles flux and energy and the dynamics of their interaction with molecules in the atmosphere.

The calculated terrestrial vertical muon flux is in excellent agreement with measurements. On Earth, the atmosphere is sufficiently thick that a cosmic ray shower maximum (the altitude at which the shower is at a maximum in terms of the number of secondary particles) is reached after $1 / 10$ of the atmosphere is traversed, or at $\sim 15 \mathrm{~km}$ altitude. Below this altitude, the net number and energy of the secondary particles decreases. The rate of generation of muons within the Earth's atmosphere is roughly 1000 per meter squared per second, with the maximum muon flux coinciding approximately with the shower maximum. The muon flux then slowly decreases as the muons pass through the additional atmospheric depth until at sea level the measured vertical count rate is 70 muons $/ \mathrm{m}^{2} / \mathrm{s} / \mathrm{sr},\left(\sim 1 \mathrm{muon} / \mathrm{cm}^{2} / \mathrm{min}\right)$.

In other planetary atmospheres, such as Mars (Gierasch and Toon, 1973) or Titan (Lopez-Moreno et al., 2008) the evolution of cosmic ray showers is different than on Earth and therefore requires a planet-specific study to determine the muon fluxes and the detector requirements. The Mars surface pressure is $\sim 1 / 100$ that of Earth, which means that on 
Table 1. Vertical flux of protons, pions and muons, integrated in energy, at the atmospheric depth corresponding to the Martian surface.

\begin{tabular}{lrrr}
\hline Depth $(\mathrm{hPa})$ & 7 & 100 & 200 \\
\hline Proton $\left(\mathrm{m}^{-2} \mathrm{~s}^{-1} \mathrm{sr}^{-1}\right)$ & 9000 & 5000 & 2000 \\
$\pi^{+/-}\left(\mathrm{m}^{-2} \mathrm{~s}^{-1} \mathrm{sr}^{-1}\right)$ & 2 & 10 & 8 \\
$\mu^{+/-}\left(\mathrm{m}^{-2} \mathrm{~s}^{-1} \mathrm{sr}^{-1}\right)$ & 40 & 200 & 300 \\
\hline
\end{tabular}

Mars many of the primary particles reach the surface before undergoing a primary interaction, so the cosmic ray showers do not reach their maximum. This results in both a lower meson production rate and a lower meson loss rate than in Earth's atmosphere. In addition, the steep power law dependence in the early stages of the shower cascade suggests that the meson flux and thus the muon flux will be strongly dependent on the atmospheric pressure, zenith angle and muon energy (Fig. 3). Although the horizontal muon rate on Earth is suppressed compared to the vertical muons due to the additional atmospheric mass through which the muons transverse, the opposite is true on Mars, where the longer horizontal traverse puts the shower maximum closer to the Mars surface, making the horizontal flux greater than the vertical muon flux. The prediction is that the integration times needed for similar muon radiography resolutions will be within a factor of $\sim 2$ shorter than those on Earth.

Unlike on Earth, a large fraction of the primary rays are likely to reach the Martian surface. Additionally, pions created by the primary rays created in hadronic interactions may not decay into muons prior to reaching the surface, and therefore a high level of non-muonic charged particle flux is expected on Mars. Although the chemical composition of the Martian atmosphere is different from Earth's, the known primary and secondary flux in the upper Earth atmosphere can be used for estimating the background rates on Mars (Berringer et al., 2012). Table 1 shows the vertical flux of protons, pions and muons, integrated in energy, at the atmospheric depth is $7 \mathrm{hPa}$, which corresponds to the Martian surface. At this depth the proton flux is $\sim 200$ times stronger than the muon flux. The pion flux is only $5 \%$ of the muon flux, but it is much higher than the ratio observed on Earth's surface $(<0.1 \%)$. Table 1 also displays the vertical flux of these particles at atmospheric depths of 100 and $200 \mathrm{hPa}$, which correspond to the total thickness of the atmosphere that the muons traverse horizontally $\left(85^{\circ}\right.$ and $89^{\circ}$ from zenith). The ratio between the proton and the muon flux is 25 and 7 at $100 \mathrm{hPa}$ and $200 \mathrm{hPa}$, respectively.

These non-muonic charged particles raining down on the Martian surface will produce an additional source of unwanted background noise as compared to Earth. Vertical random shower particles could easily be rejected by selecting only linear trajectories within multiple (5-6) layers of position sensitive detectors (PSDs), thus improving the background rejection rate. A key concern, however, is the noise
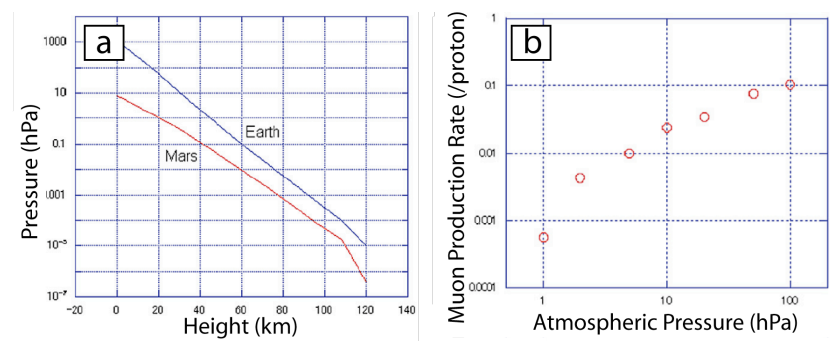

Fig. 3. From Tanka (2007). (a) Terrestrial and Martian atmospheric pressure profile. (b) Estimation of muon production rate on Mars per incident proton, integrating over the zenith from $-90^{\circ}$ to $90^{\circ}$, at Martian surface as a function of the atmospheric thickness for primaries between 1 and $20 \mathrm{GeV}$. For muons between $20 \mathrm{GeV}$ and $5 \mathrm{TeV}$ the production rate is over 10 times greater at all pressures.

level in the primary horizontal flux. As shown in Table 1, the horizontal proton flux may be much higher than the muon flux on Mars. Numerous solutions exist to remove this horizontal hadronic flux, one is utilizing both multiplicity analysis (Tanaka et al., 2001) and a hadron absorber/scatterer (burst detector). The hadronic shower produced in the hadron absorber (e.g. Pb plates) between PSDs would be rejected by discarding two or more simultaneous signals from one PSD. While this solution can be applied on the Earth's surface or on a balloon, it may be an impractical solution for a Mars-bound instrument due to the additional mass needed. A simple alternative is direct screening of the background hadronic components by using the target itself. Since the sizes of the targets of interest are large, they will provide sufficient shielding from non-muonic particles.

In the future, a more detailed Extended Air Shower Monte Carlo simulation will be necessary in order to estimate the flux of charged particles having incident angles of more than $90^{\circ}$ from zenith, and a detailed detector design simulation using GEANT (Agostinelli et al., 2003) to determine the optimum detector segmentation, layering, and timing resolution necessary to achieve the needed background suppression for the science targets of interest.

\section{A flexible, low-power instrument that will continuously collect data with minimal impact on the mission's primary instruments and operations}

The adaptation of muon detectors and instrumentation for planetary exploration offers several unique advantages (Fig. 4).

- Low power consumption: a muon detector would require only $\sim 2-3$ watts (Tanaka, 2012; Taira and Tanaka, 2010; Uchida et al., 2009) (by comparison, a fraction of the Mars Science Multi-Mission Laboratory Radioisotope Thermoelectric Generator (RTG) power production of 110 watts). Even in Martian winter when 


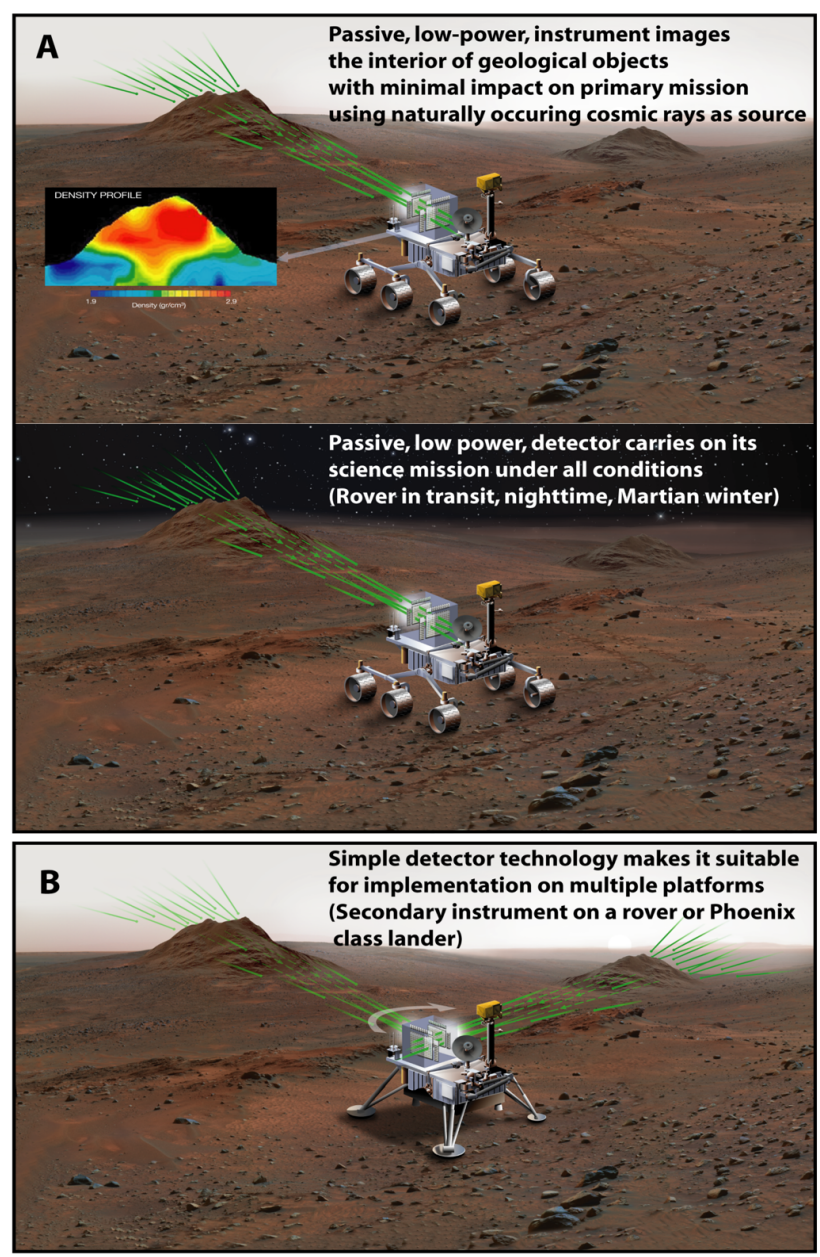

Fig. 4. Operational concept. Muons generated by interactions of primary cosmic rays in the planet's atmosphere (green spheres) pass through a geologic object of interest, and are partially absorbed by the object. A passive muon detector composed of parallel scintillating plates on a lander or a rover records the tracks of the muons. The recorded tracks are analyzed on site to determine the direction from which they entered the detector and the amount of energy absorbed by the target. The observations are distilled into a density image of the geological target, much like an X-ray radiograph would, except using muons as a passive source of radiation. Two concepts are presented: (A) Muon radiography instrument mounted as a secondary instrument on a rover. (B) The instrument is mounted on a small Phoenix class lander observing multiple targets during the life of the mission.

more power is typically routed into heating and less into science instruments, the muon radiography instrument could continue to acquire data, allowing the instrument to be in a state of perpetually acquiring science data.

- Extremely low data rates: the simple detector concept yields a single data product - a density profile image a few kilobytes in size that can be easily transmitted back to Earth.
- Flexible implementation strategy and operations at all conditions: the muon instrument is a passive detector with no moving parts, weak pointing requirements, a single operating mode, and relatively low computational and processing requirements. Thus, its impact on operations, command and control, and ground resources makes it an easily accommodated, low-impact payload. In a Mars rover configuration (Fig. 4a), the instrument could be either a primary or a secondary payload that would continuously gather data on geological targets along the rover's route. In fact, as viewing geometry varies during the rovers, accurately recorded, traverse, additional structural information would be gained on the internal structure of these targets. Large objects several kilometers in diameters that would require several weeks of muon integration can be strategically targeted for observation during periods when the rover is sedentary, whereas smaller objects $(\sim 1 \mathrm{~km}$ diameter) can be observed during routine rover operations without affecting the rover primary science mission. As was recently demonstrated by Tanaka et al. (2012), the technique was able to resolve density variations inside Mt. Omuro, Japan, using a "roving" detector. The detector was mounted in an automobile and repositioned at 18 different locations around the target volcano, collecting data for $20 \mathrm{~min}$ at each location. This rover configuration enables the instrument to image sections of the structure and function as a tomograph, eliminating the need to have detailed knowledge of the target topography. A rover configuration provides tremendous flexibility in target selection by using route optimization to trade spatial and temporal resolution for shorter integration times. Alternatively, a muon detector could also be mounted on a Discovery class small lander (Fig. 4b) and target in turn multiple geological features of interest around the landing site.

\section{Candidate geological targets on Mars}

Muon radiography has the potential to reveal the interior structure of a number of geological features on the surface of Mars, addressing key questions in geologic history, climate, biologic potential, and the nature of current activity. Six potential target geological features are shown in Fig. 5. Among the targets are glacial and periglacial features such as putative pingoes, ice-cored mounds formed by the freezing of pressurized groundwater (Dundas et al., 2008), and mid-latitude ice-masses ("lobate debris aprons", Plaut et al., 2008) that have been penetrated by sounding radar and likely represent remnants of glaciers from a different climate epoch (Plaut et al., 2008; Holt et al., 2008). Another possible target class is mesas in so-called "chaos" regions that were the source of massive outflow water floods and may currently contain confined aquifers. Volcanic structures have been imaged with the 


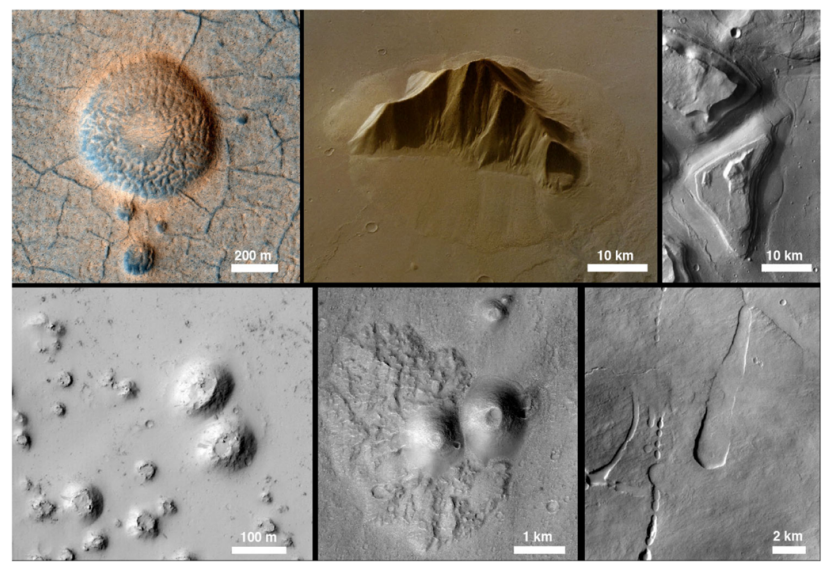

Fig. 5. Orbital images of examples of classes of potential targets for muon radiography on Mars. Top left: aputative pingo (ice-cored mound) in Utopia Planitia (HiRISE). Top middle: perspective view of a massif in the Eastern Hellas region with surrounding ice-rich lobate aprons. The target for muon radiography would be the aprons surrounding the massif, which are up to $1 \mathrm{~km}$ thick in places. (Elevation and image data from HRSC). Top right: mesas in Hydraotes Chaos, once a source of outflow flood waters, and potentially a host of remnant aquifers (THEMIS visible). Lower left: rootless cones on platy lava flows in Elysium Planitia (HiRISE). Lower middle: Enigmatic edifices in Hydraotes region that may be rootless, or connected to a vent system (HiRISE). Lower right: chains of collapse pit on Ascraeus Mons. Areas between pit chains may contain lava tubes or caves in the subsurface.

technique on Earth, and numerous features on Mars are potential targets, including small volcanic edifices associated with recent platy lava flows of Elysium Planitia that may suggest ongoing activity (Lanagan et al., 2001). The rootless cones in this region are thought to result from the interaction of lava with ground ice; imaging the interior structure could resolve questions of their origin. Larger volcanic edifices could be probed. This potentially would allow determination of the eruptive sequence of edifices, e.g., whether a structure is monogenetic, the result of multiple events, or a stratovolcano.

A geological target on the forefront of Mars exploration that is particularly suited for investigation using muon radiography is the detection and delineation of planetary caverns - the most promising environment identified to date for potential biological activity on Mars. Caves on Mars have been suggested as high priority targets for evidence of biological activity, as they afford shelter from radiation and extreme temperatures and may serve as pathways for groundwater flow (e.g., Boston et al., 2004). Cave targets on the flanks of Arsia Mons where Cushing et al. (2007) identified numerous candidates for cave skylights (Fig. 6) are a prime example of this class of targets. The lower flanks of Arsia Mons are marked by chains of collapse pits, fully collapsed channels, and broad troughs. Chains of pits are several tens

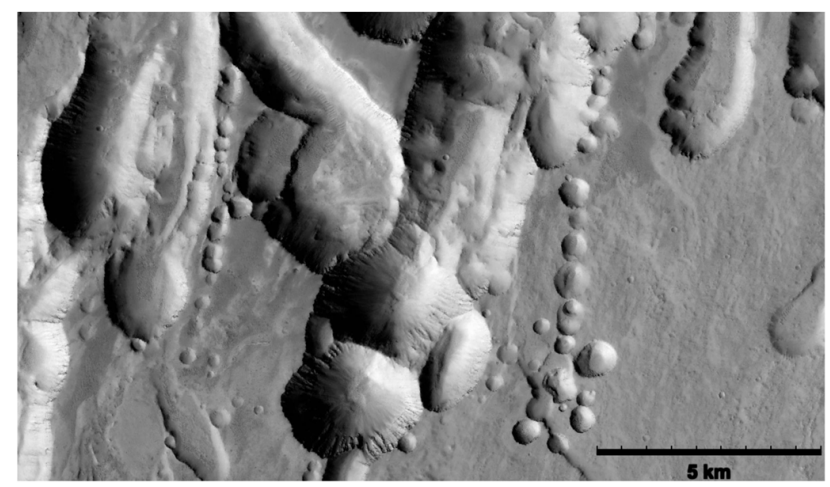

Fig. 6. Potential primary target for muon radiography exploration on Mars. Complex terrain of collapse structures on the lower flanks of Arsia Mons, Mars likely contains lava tubes and caves in the subsurface (Cushing et al., 2007; Williams et al., 2010). Muon radiography obtained from trough floors could be used to map the void space associated with partially collapsed lava tubes. High-resolution digital topography data can be used to delineate the 3-D geometry of the candidate targets including possible observation vantage points. CTX image, MSSS/JPL/NASA; $7.8^{\circ} \mathrm{S}, 240.6^{\circ} \mathrm{E}$.

of kilometers long, with individual pits up to $\sim 1 \mathrm{~km}$ in diameter and several tens of meters deep. From the floor of a trough, a rover or a lander equipped with a muon radiography system could target the subsurface in the gaps of pit chains to localize and map voids formed by lava tubes. For example, the broad troughs on the north flank of Arsia Mons (Fig. 6) are typically $500 \mathrm{~m}$ deep and $2 \mathrm{~km}$ wide. A detector positioned on the floor of a trough within $1 \mathrm{~km}$ of the trough wall would be suitably positioned to detect muons incident at angles less than $\sim 30$ degrees from the horizontal at the surface above the scarp. The high contrast in density between void space and surrounding lava rock would provide a strong signal in the muon data. A future feasibility study of the concept would have to include detailed analysis of the viewing geometry of potential targets on Mars' surface.

\section{Comparison of muon tomography to alternative technologies for shallow geology exploration}

A comparison of muon radiography with some of the obvious alternative subsurface imaging techniques, seismic tomography, ground penetrating radar (GPR), microgravimetry surveying, and magnetic surveying, suggests that it would offer several implementation and operation advantages.

Ground penetrating radar (GPR) is a method whereby the subsurface is imaged by measuring the reflection of a transmitted electromagnetic pulse off subsurface reflectors. Material properties and boundaries are determined from the timing, strength, and shape of the returned pulse, which depends on the material dielectric properties. GPR is the technology of choice for measuring surface ice thickness and properties, 
as was shown by Plaut et al. (2007) who used the MARSIS instrument on the Mars Express Orbiter, taking advantage of the near-transparency of ice to radar. However, for imaging a rocky subsurface or the interior of a geological fold, the radar, which requires a powerful transmitter and a large antenna, typically only penetrates up to $\sim 100 \mathrm{~m}$, and less if the top layers are iron rich as is the case on the Martian surface (Heggy et al., 2003). Remote, space-based GPR instruments will not adequately resolve horizontal structure of relatively small surface features. As was demonstrated by Tanaka et al. (2009a, b), muon radiography offers a clear advantage in these scenarios, thanks to the far deeper penetration depths of secondary muons. Muon detectors are also passive sensors, without the need for large antennas and power sources to generate, shape, and detect the return of high-energy beams or pulses.

Similar to GPR, seismic tomography uses elastic wave energy to map subsurface elastic material properties. Seismic tomography has been used since the early days of terrestrial seismology to map subsurface features of varying scales, from the global Earth structure to sub-kilometer crustal layering. The resolution of the technique is strongly dependent on the number of sources and receivers. Due to constraints on mission complexity, it is safe to assume that any potential seismic deployment would rely on naturally occurring seismic events (quakes, volcanic activity, meteorite impacts) rather than active sources such as explosives or vibrators as is the standard practice in terrestrial exploration geophysics. On Mars, seismicity is estimated to be a factor of $\sim 1000$ lower than on Earth (Knapmeyer et al., 2006), requiring highly sensitive, elaborate seismic stations operating for several years. Moreover, just as with GPR, the resolution of the tomographic image depends on the wavelength of the seismic waves, which is typically on the order of several hundred meters to several kilometers from natural events. As demonstrated by Tanaka et al. (2010, 2009a, b), muon radiography obtains at least an order-of-magnitude improvement in spatial resolution over these wavelengths. Seismology would be the technique of choice for studying global scale planetary features such as resolving the size of the Martian core, or the base of the Martian crust, but muon radiography offers clear advantages for studying several-kilometer scale geological features near the surface, and small geological features such as cavernous structures.

Two other techniques commonly used on Earth are microgravimetry surveying and magnetic surveying. Both techniques are passive and are routinely used on Earth for oil and mineral exploration. A key advantage they have over muon tomography is the ability to look downward into the Earth, whereas muon tomography requires special geometry enabling muons to path through the atmosphere and the target prior to reaching the detector. The main challenge both techniques pose for Mars exploration is that they require being physically near the target area; this may not be possible with the topography of some features. This may not necessarily be

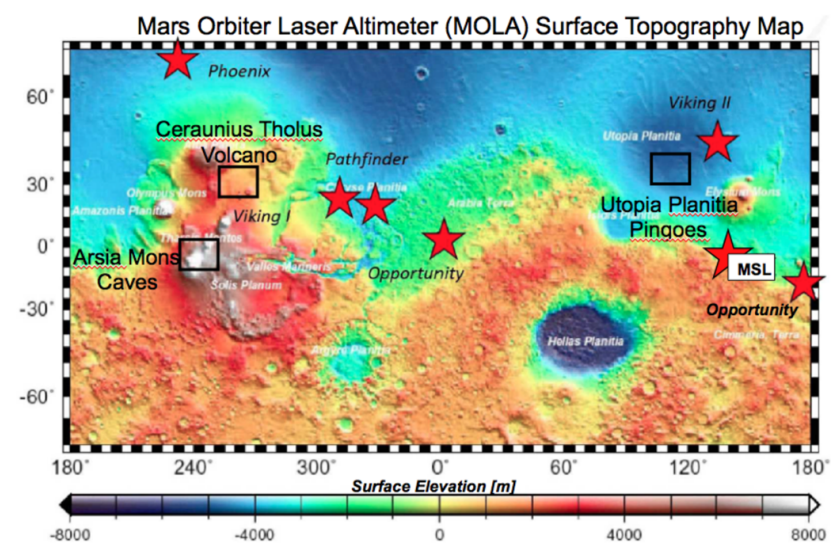

Fig. 7. An elevation map showing some of the geological regions of interest (black rectangles) and locations of past Mars landers (red stars) from the Mars Orbiter Laser Altimeter MOLA (Smith et al., 1999).

possible on Mars, and will place strong constraints on a future rover mission. Muon tomography, by comparison, may be able to survey targets from a distance, and may offer the flexibility of observing targets of opportunity from a rover without impacting the rover's primary mission. Nevertheless, muon radiography can be implemented in concert with microgravimeters and magnetometers to obtain independent and complementary observations of a geological target.

\section{Challenges for muon tomography on Mars}

There are several challenges in developing a muon tomography instrument for Mars. One key challenge is reducing the detector mass. The detectors that have been deployed on Earth for studying large $(100 \mathrm{~m}-2 \mathrm{~km})$ geological features are free of the mass constraints that are a critical consideration in any Mars lander design. There are several ways of reducing the overall detector mass, from reducing its overall size, reducing the scintillator bars thickness, or seeking an alternative detector technology. In the design of a Mars muon telescope the trades between such options and their implications on detector electronics, the scientific requirements on target resolution, and integration times will have to be carefully considered.

Equally important are power considerations. Muon tomography is, in principle, a low power technology as it does not depend on an active power source. As has been demonstrated by Tanaka (2012) and Tanaka et al. (2010, 2009a,b, 2008) it can be implemented with relatively low (several watts) of power. Yet, the power demands of an extended Mars mission would almost certainly require a re-examination of the power constraints in the context of a specific mission.

Another key challenge is developing a detector that can survive the physically demanding phase of Entry, Descent, and Landing (EDL) that is a critical part of every Mars 
surface mission, in which the lander is exposed to large accelerations, shocks and vibrations. Moreover, it is likely that detector planes may be required to be stowed in a folded position for EDL and then deployed into a telescope configuration once on the planet's surface. This would add mechanical complexity, with associated additional mass and power requirements.

Environmental challenges on the Martian surface would require that the detector and detector electronics operate at low temperatures. Mars average temperature is below $-50^{\circ} \mathrm{C}$, and depending on the landing environment, will be exposed to extreme (tens of ${ }^{\circ} \mathrm{C}$ ) temperature variations. Moreover, shielding from radiation by the thin Mars atmosphere would be significantly weaker than on Earth's surface and could result in quicker degradation of detector performance. Extensive laboratory testing of detector performance of Martian conditions will be essential.

Finally, choosing a landing site for Martian muon tomography poses its own inherent challenges. Some of the more interesting geological targets on the Martian surface are at high altitude where landing is more difficult (Fig. 7). Moreover, target viewing geometry may dictate precise landing within a few kilometers of the object of interest. The recent success of the Mars Science Laboratory (MSL) (Grotzinger et al., 2012) has benefitted from advances in both landing technology and precision landing that can help answer these challenges.

\section{Summary}

Muon telescopes represent a new potential class of instruments for planetary exploration, providing a new type of measurement for the detection and delineation of potentially habitable subsurface environments, and for the interpretation of composition and evolutionary state of the Martian surface. Muon radiography is a proven, simple, low cost (muon detectors are routinely built in physics laboratories worldwide), and efficient technology that could detect subsurface radiation-shielded habitable environments such as lava tubes or caverns that might not be detectable by any other technique available today. Thanks to low power and data rate demands, it could be integrated as a secondary instrument on future missions with minimal impact on primary mission operations. A mission that includes a muon detector could set the stage for a future mission to directly explore subsurface habitable environments on Mars. Landing and operating a muon detector on Mars pose challenges that have yet to be encountered in terrestrial applications of the technology. Nevertheless, these challenges are understood, and mainly involve detector design and engineering. Moreover, recent advances in navigation and EDL technology as demonstrated by MSL offer hope that in the future a muon telescope could be landed in the vicinity of high-scientific-value geological targets on Mars.
Acknowledgements. The authors wish to thank the Associate for the thoughtful and constructive critique of the manuscript. This research was conducted in part at the Jet Propulsion Laboratory, California Institute of Technology, under contract with the National Aeronautics and Space Administration.

Edited by: J. Albert

\section{References}

Agostinelli, S., Allison, J., Amako, K., Apostolakis, J., Araujo, H., Arce, P., Asai, M., Axen, D., Banerjee, S., Barrand, G., Behner, F., Bellagamba, L., Boudreau, J., Broglia, L., Brunengo, A., Burkhardt, H., Chauvie, S., Chuma, J., Chytracek, R., Cooperman, G., Cosmo, G., Degtyarenko, P., dell'Acqua, A., Depaola, G., Dietrich, D., Enami, R., Feliciello, A., Ferguson, C., Fesefeldt, H., Folger, G., Foppiano, F., Forti, A., Garelli, S., Giani, S., Giannitrapani, R., Gibin, D., Gómez Cadenas, J. J., González, I., Gracia Abril, G., Greeniaus, G., Greiner, W., Grichine, V., Grossheim, A., Guatelli, S., Gumplinger, P., Hamatsu, R., Hashimoto, K., Hasui, H., Heikkinen, A., Howard, A., Ivanchenko, V., Johnson, A., Jones, F. W., Kallenbach, J., Kanaya, N., Kawabata, M., Kawabata, Y., Kawaguti, M., Kelner, S., Kent, P., Kimura, A., Kodama, T., Kokoulin, R., Kossov, M., Kurashige, H., Lamanna, E., Lampén, T., Lara, V., Lefebure, V., Lei, F., Liendl, M., Lockman, W., Longo, F., Magni, S., Maire, M., Medernach, E., Minamimoto, K., Mora de Freitas, P., Morita, Y., Murakami, K., Nagamatu, M., Nartallo, R., Nieminen, P., Nishimura, T., Ohtsubo, K., Okamura, M., O’Neale, S., Oohata, Y., Paech, K., Perl, J., Pfeiffer, A., Pia, M. G., Ranjard, F., Rybin, A., Sadilov, S., di Salvo, E., Santin, G., Sasaki, T., Savvas, N., Sawada, Y., Scherer, S., Sei, S., Sirotenko, V., Smith, D., Starkov, N., Stoecker, H., Sulkimo, J., Takahata, M., Tanaka, S., Tcherniaev, E., Safai Tehrani, E., Tropeano, M., Truscott, P., Uno, H., Urban, L., Urban, P., Verderi, M., Walkden, A., Wander, W., Weber, H., Wellisch, J. P., Wenaus, T., Williams, D. C., Wright, D., Yamada, T., Yoshida, H., and Zschiesche, D.: Geant4 - a simulation toolkit, Nucl. Instrum. Meth. A, 506, 270-278, 2003.

Alvarez, L. W., Anderson, J. A., El Bedwei, F., Burkhard, J., Fakhry, A., Girgis, A., Goneid, A., Hassan, F., Iverson, D., Lynch, G., Miligy, Z., Moussa, A. H., Sharkawi, M., and Yazolino, L.: Search for Hidden Chambers in the Pyramids, Science, 167, 832839, 1970.

Anderson, E. W., Lindsey, C. S., Wang, C. H., Hojvat, C., Reeves, D., and Turkot, F.: A scintillator hodoscope at the tevatron collider, Nucl. Instrum. Meth. A, 295, 86-93, 1990.

Berringer, J., Arguin, J.-F., Barnett, R. M. et al.: Particle Data Group, Phys. Rev. D, 86, 010001, 2012.

Boston, P. J., Frederick, R. D., Welch, S. M., Werker, J., Meyer, T. R., Sprungman, B., Hildreth-Werker, V., and Thompson, S. L.: Extraterrestrial Subsurface Technology Test Bed: Human Use and Scientific Value of Martian Caves Space Tech. \& Applic. Forum 2003 Bull. AIP \#654, Amer. Inst. of Physics, College Park, MD, 2004.

Cushing, G. E., Titus, T. N., Wynne, J. J., and Christensen, P. R.: THEMIS observes possible cave skylights on Mars, Geophys. Res. Lett., 34, L17201, doi:10.1029/2007GL030709, 2007. 
Dundas, C. M., Mellon, M. T., McEwen, A. S., Lefort, A., Keszthelyi, L. P., and Thomas, N.: HiRISE observations of fractured mounds: Possible Martian pingos, Geophys. Res. Lett., 35, L04201, doi:10.1029/2007GL031798, 2008.

Flaser, F. M.: The Dynamic Meteorology of Titian, Planet. Space Sci., 46, 1125-1147, 1998.

Gaisser, T. K.: Cosmic Rays and Particle Physics, Cambridge University Press ISBN 0-521-33931-6, 1990.

George, E. P.: Cosmic Rays Measure Overburden of Tunnel, Commonwealth Engineer, 455-457, 1955.

Gierasch, P. J. and Toon, O. B.: Atmospheric Pressure Variation and the Climate of Mars, J. Atmos. Sci., 30, 1502-1508, 1973.

Grieder, P. K. F.: Cosmic Rays At Earth, Elsevier, ISBN 0-44450710-8, 2001.

Grotzinger, J. P., Crisp, J., Vasavada, A. R., Anderson, R. C., Baker, C. J., Barry, R., Blake, D. F., Conrad, P., Edgett, K. S., Ferdowski, B., Gellert, R., Gilbert, J. B., Golombek, M., Gómez-Elvira, J., Hassler, D. M., Jandura, L., Litvak, M., Mahaffy, P., Maki, J., Meyer, M., Malin, M. C., Mitrofanov, I., Simmonds, J. J., Vaniman, D., Welch, R. V., and Wiens, R. C.: Mars Science Laboratory Mission and Science Investigation, Space Sci. Rev., 170, 5-56, 2012.

Heggy, E., Paillou, P., Costard, F., Mangold, N., Ruffie, G., Demontoux, F., Grandjean, G., and Malezieux, J. M.: Local Geoelectrical Models of the Martian Subsurface for Shallow Groundwater Detection Using Sounding Radars, J. Geophys. Res., 108, 8030, doi:10.1029/2002JE001871, 2003.

Holt, J. W., Safaeinili, A., Plaut, J. J., Head, J. W., Phillips, R. J., Seu, R., Kempf, S. D., Choudhary, P., Young, D. A., Putzig, N. E., Biccari, D., and Gim, Y.: Radar sounding evidence for buried glaciers in the southern mid-latitudes of Mars, Science, 322, 1235, doi:10.1126/science.1164246, 2008.

Knapmeyer, M., Oberst, J., Hauber, E., Wahlisch, M., Deuchler, C., and Wagner R.: Working Models for Spatial Distribution and Level of Mars' Seismicity, J. Geophys. Res., 111, E11006, doi:10.1029/2006JE002708, 2006.

Lanagan, P. D., McEwen, A. S., Keszthelyi, L. P., and Thordarson, T.: Rootless cones on Mars indicating the presence of shallow equatorial ground ice in recent times, Geophys. Res. Lett., 28, 2365-2367, 2001.

Lopez-Moreno, J. J., Molina-Cuberos, J. J., Hamelin, M., Grard, R., Simoes, F., Godard, R., Schwingenschuh, K., Beghin, C., Berthelier, J. J., Brown, V. J. G., Falkner, P., Ferri, F., Fulchignoni, M., Jernej, I., Jeronimo, J. M., Rodrigo, R., and Trautner, R.: Structure of Titan's low altitude ionized layer from the Relaxation Probe onboard HUYGENS, Geophys. Res. Lett., 35, L22104, doi:10.1029/2008GL035338, 2008.

Plaut, J. J., Picardi, G. Safaeinili, A., Ivanov, A., Milkovich, S. M., Cicchetti, A., Kofman, W., Mouginot, J., Farrell, W. M., Phillips, R. J., Clifford, S. M., Frigeri, A., Orosei, R., Federico, C., Williams, I. P., Gurnett, D. A., Nielsen, E., Hagfors, T., Heggy, E., Stofan, E. R., Plettemeier, D., Watters, T. R., Leuschen, C. J., and Edenhofer, P.: Subsurface Radar Sounding of the South Polar Layered Deposits of Mars, Science, 316, 9295, doi:10.1126/science.1139672, 2007.
Plaut, J. J., Safaeinili, A., Holt, J. W., Phillips, R. J., Head, J. W., Seu, R., Putzig, N. E., and Frigeri, A.: Radar evidence for ice in lobate debris aprons in the mid-northern latitudes of Mars, Geophys. Res. Lett., 36, L02203, doi:10.1029/2008GL036379, 2008.

Reichenbacher, J. and De Jong, J.: Calculation of the Underground Muon Intensity Crouch Curve from a Parameterization of the Flux at Surface, 30th International Cosmic Ray Conference (ICRC07), 2007.

Smith, D. E., Zuber, M. T., Solomon, S. C., Phillips, R. J., Head, J. W., Garvin, J. B., Banerdt, W. B., Muhleman, D. O., Pettengill, G. H., Neumann, G. A., Lemoine, F. G., Abshire, J. B., Aharonson, O., Brown, C. D., Hauck, S. A., Ivanov, A. B., McGovern, P. J., Zwally, H. J., and Duxbury, T. C.: The global topography of Mars and implications for surface evolution, Science, 284, 5419, doi:10.1126/science.284.5419.1495, 1999.

Taira, H. and Tanaka, H. K. M.: A potential space- and power - effective muon sensor module for imaging a volcano, Earth Planet. Space, 62, 179-186, 2010.

Tanaka, H.: Monte-Carlo simulations of atmospheric muon production: Implication of the past martian environment, Icarus, 191, 603-615, 2007.

Tanaka, H. K. M.: Volume slicing with mulit-directional muon radiography, International Workshop on "Muon and Neutrino Radiography", 17-20 April, 2012.

Tanaka, H., Nagamine, K., Kawamura, N., Nakamura, S. N., Ishida, K., and Shimomura, K.: Development of the cosmic-ray muon detection system for probing internal-structure of a volcano, Hyperfine Interact., 138, 521-526, 2001.

Tanaka, H., Nakano, T., Takahashi, S., Yoshida, J., Takeo, M., Ohminato, T., Aoki, Y., Koyama, E., Tsuji, H., Ohshima, H., Maekawa, T., Watanabe, H., and Niwa, K.: Radiographic Imaging Below a Volcanic Crater Floor With Cosmic-Ray Muons, Am. J. Sci., 308, 843-850, 2008.

Tanaka, H., Uchida, T., Tanaka, M., Shinohara, H., and Taira, H.: Cosmic-Ray Muon Imaging of Magma in a Conduit: Degassing Process of Satsuma-Iwojima Volcano, Japan, Geophys. Res. Lett., 36, L01304, doi:10.1029/2008GL036451, 2009a.

Tanaka, H., Uchida, T., Tanaka, M., Takeo, M., Oikawa, J., Ohminato, T., Nakano, T., Aoki, Y., Koyama, E., and Tsuji, H.: Detecting a Mass Change Inside a Volcano by Cosmic-Ray Muon Radiography (Muography): First Results from Measurements at Asama Volcano, Japan, Geophys. Res. Lett., 36, L17302, doi:10.1029/2009GL039448, 2009b.

Tanaka, H. K. M., Taira, H., Uchida, T., Tanaka, M., Takeo, M., Ohiminato, T., and Tsuji, H.: Three-dimensional computational axial tomography scan of a volcano with cosmic ray muon radiograph, J. Geophys. Res., 115, B12332, doi:10.1029/2010JB007677, 2010.

Uchida, T., Tanaka, H. K. M., and Tanaka, M.: Space Saving and Power Efficient Readout System for Cosmic-Ray Muon Radiography, IEEE T. Nucl. Sci., 56, 448-452, 2009.

Williams, K. E., McKay, C. P., Toon, O. B., and Head, J. W.: Do Ice Caves exist on Mars?, Icarus, 209, 358-368, doi:10.1016/j.icarus.2010.03.039, 2010. 